\title{
Acidified Blue Ink-staining Procedure for the Observation of Fungal Structures Inside Roots of Two Disparate Plant Lineages \\ Jill Kowal ${ }^{*}$, Elena Arrigoni” ${ }^{\#}$ and Sophie Lane ${ }^{\#}$
}

\author{
Comparative Plant and Fungal Biology, Jodrell Laboratory, Royal Botanic Gardens, Kew, London, \\ England TW9 3AB, UK \\ *For correspondence: J.Kowal@kew.org \\ ${ }^{\#}$ Contributed equally to this work
}

\begin{abstract}
[Abstract] Identifying microscopic mycorrhizal fungal structures in roots, i.e., hyphae, vesicles and arbuscules, requires root staining procedures that are often time consuming and involves chemicals known to present health risks from exposure. By modifying established protocols, our root staining method stains roots using a safe ink- and vinegar-based staining solution, followed by a 2-16 h-long destaining period. The entire procedure can be completed in less than $6 \mathrm{~h}$ (plus up to $16 \mathrm{~h}$ de-staining overnight) and roots are suitable for semi-permanent and permanent slide mounting for light microscopy. We tested our method on hundreds of wild-sourced roots from two different plant species: Lycopodiella inundata, a herbaceous clubmoss with tough water-resistant roots, and Sambucus nigra, a temperate woody shrub. Both plants associate with endomycorrhizae, L. inundata predominantly with Mucoromycotina fine root endophytes (MucFRE) and S. nigra with Glomeromycota arbuscular mycorrhizal fungi (AMF). Here we describe a simple, efficient, repeatable and safe method to detect the presence of fungal structures using light microscopy.
\end{abstract}

Keywords: Fine root endophytes, Glomeromycota, Hyphae, Ink, Lycopodiella inundata, Mycorrhizal fungi, Mucoromycotina, Sambucus nigra, Vinegar

[Background] Here we detail an efficient and safe staining method used to analyse 1,305 Lycopodiella inundata (Figure 1A) roots for fine root endophytes (FRE) (Kowal et al., 2020) and 144 Sambucus nigra roots for coarse or AMF. We also describe how to clean roots for staining and prepare semi-permanent slides to analyse the presence/absence of these two widespread mycorrhizas present in host plant roots across the land plant phylogeny.

Established staining methods for identifying mycorrhizal fungal structures typically used trypan blue or chlorazol black E dyes (Phillips and Hayman, 1970; Agerer, 1991; Brundrett et al., 1994), understood to be carcinogenic. Other researchers described a safer alternative using an ink-vinegar staining solution and clearing with $\mathrm{KOH}$ (Vierheilig et al., 1998 and 2005; Wilkes et al., 2019) and compared techniques (Vierheilig et al., 2005), but none tested these techniques with significantly finer FRE (hyphae $<2 \mu \mathrm{m}$ wide) as observed in Lycopodiella inundata, a lycopod. We demonstrate this same method is reliable and repeatable in host plant from a later evolved angiosperm lineage, Sambucus nigra, which is colonised by wider coarse hyphae and AMF. 


\section{Materials and Reagents}

A. Root cleaning

1. Root material/plant

2. Ethanol (EtOH) (Fisher Scientific)

3. Distilled water $\left(\mathrm{dH}_{2} \mathrm{O}\right)$

B. Root clearing and staining

1. Non-filter pipette tips (SARSTEDT)

2. $2 \mathrm{ml}$ microcentrifuge tubes (Eppendorf)

3. Distilled water $\left(\mathrm{dH}_{2} \mathrm{O}\right)$

4. Blue ink (Sheaffer, catalog number: 94221)

5. Glycerol (Sigma-Aldrich)

6. Glacial acetic acid (Sigma-Aldrich)

7. Acetic acid $\left(\mathrm{C}_{2} \mathrm{H}_{4} \mathrm{O}_{2}\right)$ (Sigma-Aldrich)

8. Lactic acid (Sigma-Aldrich)

9. Potassium hydroxide $(\mathrm{KOH})$ (Fisher Scientific)

10. Staining solution-10\% Sheaffer blue ink in $25 \%$ glacial acetic acid (see Recipes)

11. De-staining solution $1-1 \%$ acetic acid (see Recipes)

12. De-staining solution $2-50 \%$ glycerol (see Recipes)

C. Microscopy

1. Glass slides $76 \times 26 \mathrm{~mm} / 0.8-1.0 \mathrm{~mm}$ (Thermo Fisher Scientific) and cover slips $18 \times 18$ $\mathrm{mm} / 0.16-0.19 \mathrm{~mm}$ (VWR)

2. Clear nail polish

3. Mounting solution-50\% glycerol for semi-permanent slides, or PVLG (Polyvinyl-Lacto-Glycerol) for permanent slides (see Recipes for both)

\section{Equipment}

A. Root cleaning

1. Fridge or cool box

2. Wash bottle

3. Paint brush

4. Small plastic dish with shallow sides

5. Airtight vessels for storing cleaned roots (Falcon tubes or glass jars are also suitable)

6. Blunt forceps 
B. Root clearing and staining

1. Scalpel blade

2. Blunt forceps

3. Wash bottle

4. 2 microcentrifuge tube racks ( 1 of which should be heat proof)

5. Water bath (we used Nickel-Electro Ltd-Clifton)

6. Washing tray containing individual shallow wells (can be glass, plastic, metal or ceramic)

7. Plastic tray

8. Pipettes

C. Microscopy

1. Stereomicroscope (Leica, model: MZ6) with halogen cold light source (Volpi, model: Intralux4001). Objectives required: 10x, 20x, 40x, 63x (MucFRE only)

2. Light microscope (Leica, model: DMLB) coupled to a camera (Leica, model: DMC5400)

3. Sewing needle or pin

4. Warming oven (only if PVLG mounting solution used for permanent slides)

\section{Software}

1. Leica Application Suite (LAS) imaging software version 4.13 (Leica)

\section{Procedure}

A. Root cleaning

1. To extract the roots from the plant (Figure 1A), rinse the roots with water to loosen and remove substrate. Maintain foliage until the identity of the fungal host plant and its root system has been verified. Gently separate the fungal host plant from intertwining root systems and avoid severing the roots (Figure 1B). 


\section{bio-protocol

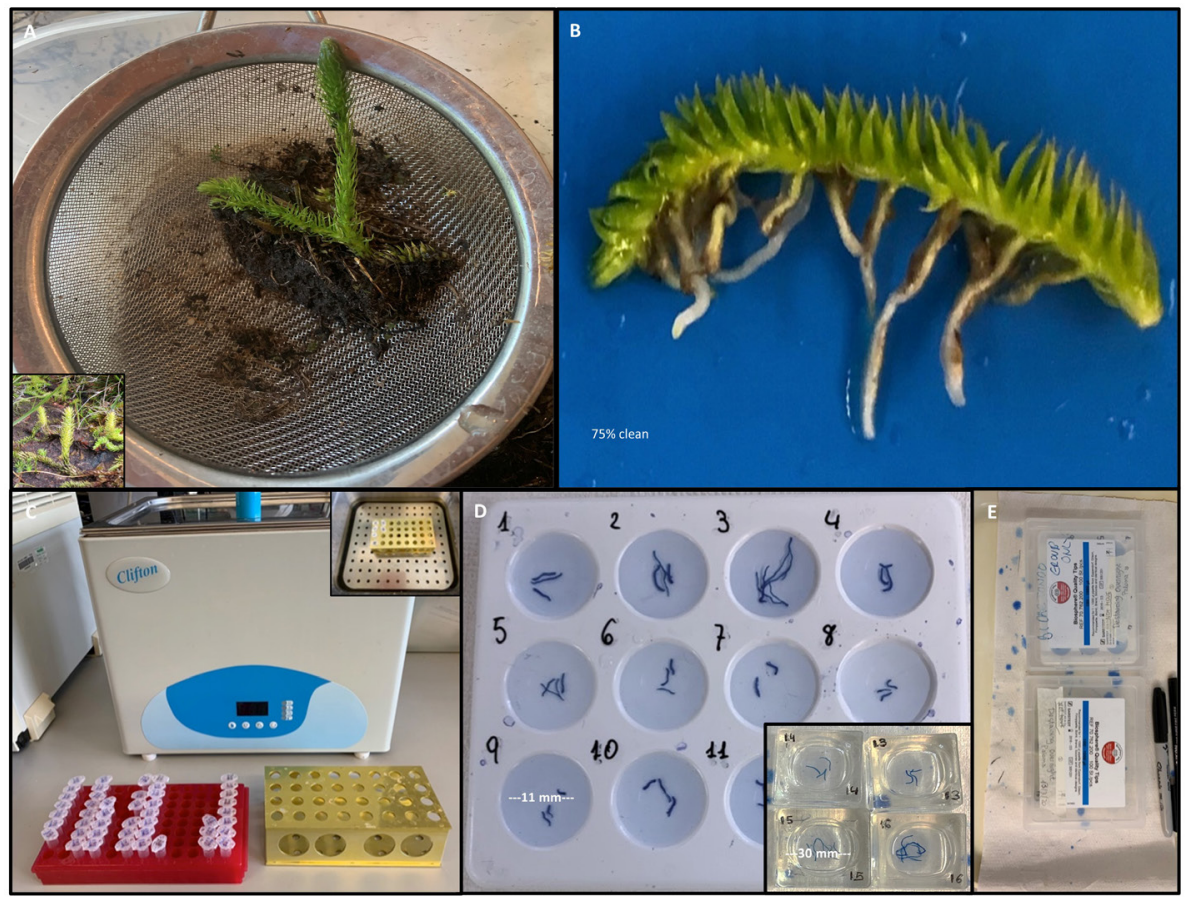

Figure 1. Images showing wild-collected Lycopodiella inundata to root staining. A. Mature field collected $L$. inundata plant (inset) shown with its soil substrate in sieve before cleaning. B. Entire L. inundata plant rhizome (average $4 \mathrm{~cm}$ long) with intact roots separated from soil substrate and $75 \%$ clean. C. Microcentrifuge tubes $(2 \mathrm{ml}$ ) set in racks for water bath (inset: tubes in metal heat-resistant rack). D. Examples of a staining plate and glass staining blocks with individual wells (inset). E. Covered staining plates using pipette container lids.

2. Place individual plant or root system into a small plastic dish and moisten with $\mathrm{dH}_{2} \mathrm{O}$; keep moist at all times.

3. Gently brush substrate off the root surfaces with a paintbrush, using a stereomicroscope and blunt forceps to hold the plant in place; avoid coarse bristles and harsh scrubbing to preserve root hair and epidermal cells.

4. Refresh water in the dish, as needed, and rinse the roots with $\mathrm{dH}_{2} \mathrm{O}$ using the wash bottle nozzle to alleviate the substrate until no debris remains (Figure 1B).

5. Once cleaned, store roots in an airtight vessel submerged in $70 \%$ ethanol for up to 1 year at room temperature, or for short-term storage in $\mathrm{dH}_{2} \mathrm{O}$ at $3^{\circ} \mathrm{C}$.

B. Preparation for staining

1. Pre-heat the water bath to $100{ }^{\circ} \mathrm{C}$, with the lid closed to accelerate heating and to avoid evaporation.

2. Cut cleaned root samples into pieces of approximately $1 \mathrm{~cm}$ in length (for ease of slide preparation later) using a sharp scalpel blade. Handle roots with blunt forceps to avoid damaging tissue.

3. For roots stored in $\mathrm{dH}_{2} \mathrm{O}$, proceed to 'Clearing' (Procedure $\mathrm{C}$ ). For roots stored in ethanol, place 
2-3 root segments into each well of the washing plate or block (Figure 1D).

4. Rinse the roots $3 \mathrm{x}$ in $\mathrm{dH}_{2} \mathrm{O}$, ensuring the roots are fully submerged.

C. Root clearing

1. Transfer the roots to (sample) labelled microcentrifuge tubes (2-3 roots per tube) in a microcentrifuge tube rack to commence clearing (Figure 1C).

2. Pipette $10 \% \mathrm{KOH}$ into the microcentrifuge tubes, submerging the roots (approximately $1 \mathrm{ml}$ ).

3. Transfer these tubes to a heat-proof microcentrifuge rack and place inside the water bath (see Figure $1 \mathrm{C}$ inset). Place a plastic tray on top to ensure tube lids do not open during boiling.

4. Boil for 15-25 min according to root thickness (see notes 5, 6 and 7).

5. Reduce the temperature to $60^{\circ} \mathrm{C}$ (this should take around 15-20 min with lid off) and leave the tubes incubating (a further $15 \mathrm{~min}$ for $S$. nigra and $30 \mathrm{~min}$ for $L$. inundata).

Carefully transfer the tubes from the water bath to the bench-top microcentrifuge tube rack.

6. Remove the $\mathrm{KOH}$ solution from root samples using a pipette, and rinse $3 \times$ with $\mathrm{H}_{2} \mathrm{O}$.

D. Staining

1. Add staining solution to the staining samples, ensuring they are fully submerged and not sticking together.

2. L. inundata: Place the staining samples in the water bath at $100{ }^{\circ} \mathrm{C}$ for $3 \mathrm{~min}$.

S. nigra: Leave the tubes at room temperature for $1 \mathrm{~min} 30 \mathrm{~s}$ (see notes 5,6 and 7 ).

E. De-staining

1. Transfer the roots to the washing tray (Figure 1D) using blunt forceps and add approximately $1 \mathrm{ml}$ de-staining solution 1 (see below, $1 \%$ acetic acid) to each well to remove excess dye. Continue administering the $1 \%$ acetic acid until the blue dye trail disappears (see notes 5, 6 and 7).

2. On the final acetic acid wash, ensure all roots are submerged and separated from each other. To prevent evaporation, place a cover over the washing tray (Figure 1E).

3. Leave to de-stain overnight.

4. Replace the $1 \%$ acetic acid with de-staining solution 2 ( $50 \%$ glycerol)-enough volume to cover the roots.

F. Slide preparation

1. To prepare a semi-permanent slide, add a drop of $50 \%$ glycerol centrally to the slide. Each cover slip should contain only one to two root segments.

2. Transfer one to two root segments to the glycerol pools using blunt forceps.

3. Position the roots as flat as possible on the slide then use a needle and stereomicroscope to centre the root and fan out the finer tips (see note 4).

4. Gently place a glass cover slip over the root segment, avoiding the creation of air bubbles. 
5. Add a drop of clear nail polish to each corner of the cover slip (to prevent root samples from drying out), and wait for this to dry before sealing around the edges.

G. Imaging

See Figure 2 for light microscopy images using this technique.

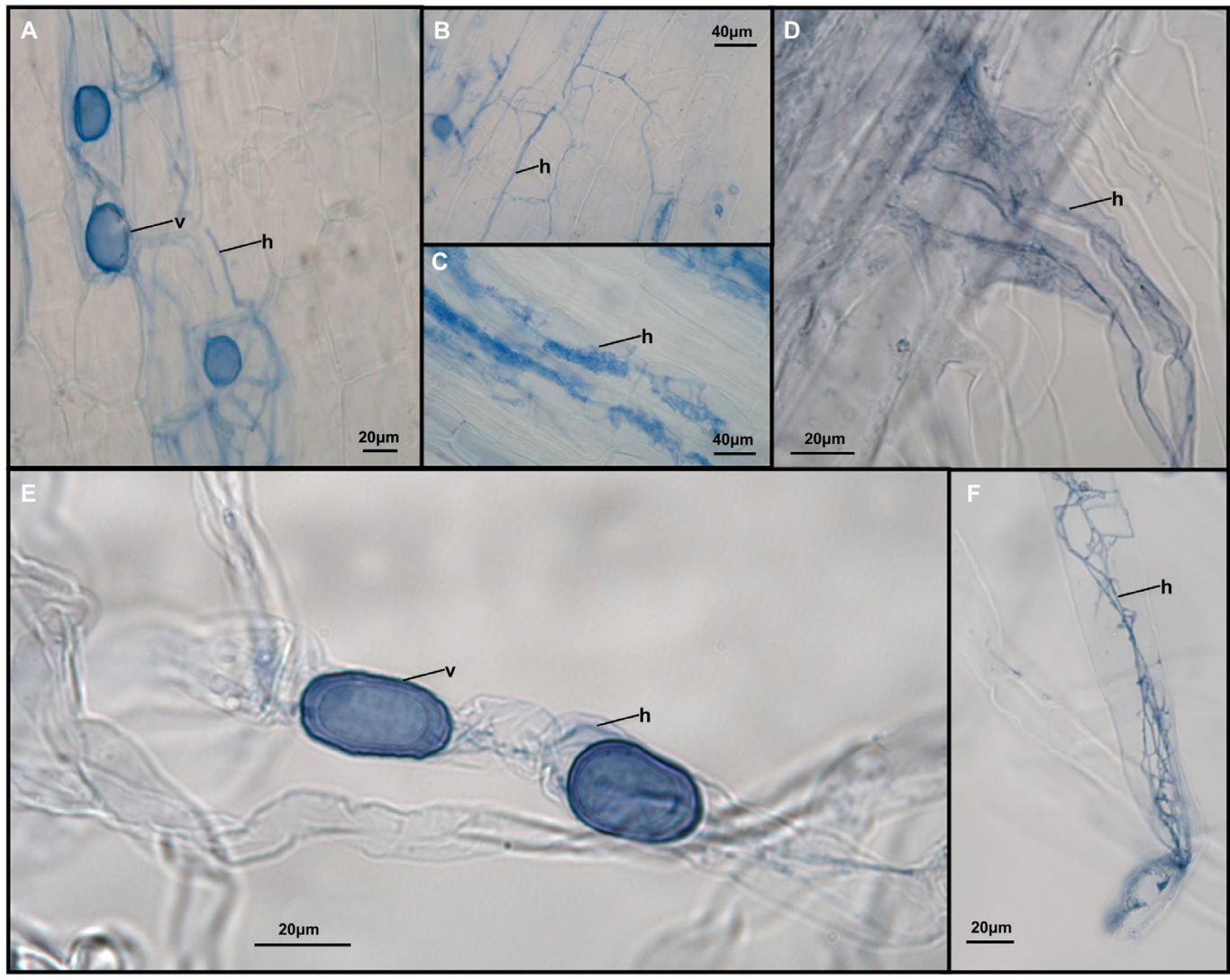

Figure 2. Micrographs of acidified Sheaffer blue ink-stained roots. Sambucus nigra: A/B. aseptate arbuscular mycorrhizal (AMF)-like intracellular hyphae (h) and vesicles (v). C. Coarse septate hyphae forming intracellular knotted clusters. Lycopodiella inundata: D. Detail of colonised root hair cells showing fine root endophytes (FRE) hyphae entering the epidermal cells. E. Pair of large vesicles in root hair cell. F. Root hair cell colonised by Mucoromycotina-like FRE. Micrographs were prepared using Leica Application Suite (LAS) imaging software version 4.13 (Leica) using a light microscope coupled to a camera. Objectives required: 40x and 63x (required for FRE only).

\section{$\underline{\text { Notes }}$}

1. Store plants (with their attached substrate) (Figure $1 \mathrm{~A}$ ) in a refrigerator at $3^{\circ} \mathrm{C}$ (if possible) and process samples within 3 days.

2. Preparation for staining: Select a range of root diameters and ages if possible.

3. De-staining: Although fungal structures are typically visible after $2 \mathrm{~h}$-we recommend leaving overnight to enhance resolution. 
4. Slide preparation:

a. For thick or woody roots, it may be necessary to flatten the sample tissue for the cover slip to adhere. In this case, gently tap the cover slip with the blunt forceps to flatten the tissue. The glycerol can be topped up under the cover slip via capillary action, if required.

b. To prepare a permanent slide-a few drops of PVLG mounting solution can be used in place of glycerol at Step F1. Once cover slips are on, these slides need to set in a $60{ }^{\circ} \mathrm{C}$ oven overnight before they can be sealed with nail polish.

5. The following parameters from this protocol are expected to vary slightly between species and sometimes within species collected from different sites:

a. Clearing time: $15-25$ min.

b. Staining temperature: $100{ }^{\circ} \mathrm{C}$ or room temperature.

c. Staining time: 1-3 min.

d. Number of de-staining washes/de-staining time (in $50 \%$ glycerol).

6. The parameters for optimum staining will vary depending on the root (e.g., age, thickness, woodiness, species of plant). For example, young roots are thinner and become easily saturated with dye-requiring shorter staining times, and fragile root samples may disintegrate if boiled during staining. Also, woody roots may need a greater number of glycerol washes (or longer washes) to de-stain fully, compared to supple roots.

7. If surplus material is available, before starting we recommend testing roots of similar condition and size to gauge optimum settings $(15,20$ or 25 min boiling time for the clearing step, and room temperature or $100{ }^{\circ} \mathrm{C}$ for staining-at 1,2 or $3 \mathrm{~min}$ ). For de-staining it is necessary to judge on a root-by-root basis whether more glycerol washes are needed, based on the amount of dye bleeding out and darkness of the roots in comparison to others.

8. For examples of stained physiological structures which can assist with the visualisation of arbuscular mycorrhizal fungi and dark septate endophytes inside root tissue, we recommend referring to the work of Priyadharsini et al., 2012 and Thangavelu and Muthuraja, 2016.

9. Store all solutions at room temperature and use within 1 month.

\section{$\underline{\text { Recipes }}$}

1. Staining solution

a. Prepare a stock solution of $25 \%$ glacial acetic acid by adding $125 \mathrm{ml}$ glacial acetic acid to $500 \mathrm{ml} \mathrm{dH} \mathrm{H}_{2} \mathrm{O}$

b. Add $25 \mathrm{ml}$ Sheaffer blue ink to $225 \mathrm{ml} 25 \%$ glacial acetic acid to prepare $10 \%$ Sheaffer blue ink, mix well

2. De-staining solutions

a. De-staining solution 1: $1 \%$ acetic acid (1:99)

Add $2.5 \mathrm{ml}$ acetic acid to $247.5 \mathrm{ml} \mathrm{dH}_{2} \mathrm{O}$

b. De-staining solution 2: $50 \%$ glycerol $(1: 1)$ 


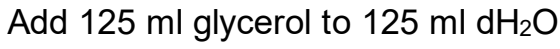

Shake repeatedly to mix well

3. Mounting solutions

a. $50 \%$ Glycerol (as above)

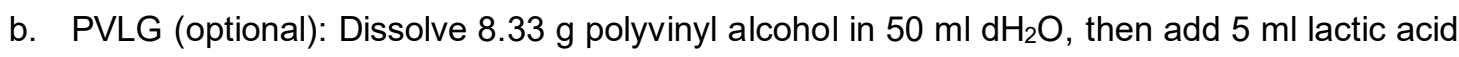
and $5 \mathrm{ml}$ glycerol, mix well (as previously described by Omar et al., 1978)

\section{Acknowledgments}

We would like to acknowledge the support of laboratory staff at the Royal Botanic Gardens, Kew.

\section{Competing interests}

The authors have no competing interests.

\section{$\underline{\text { References }}$}

1. Agerer, A. (1991). Characterization of Ectomycorrhiza. In: Norris, J. R., Read, D. J. and Varma, A.K. (Eds.). Methods in Microbiology, vol 23, pp. 25-73, London, England: Academic Press Limited.

2. Brundrett, M.C., Melville, L. and Peterson, R.L. (1994). Practical methods in mycorrhizal research. Waterloo, Canada: Mycologue Publications.

3. Kowal, J., Arrigoni, E., Serra, J. and Bidartondo, M. (2020). Prevalence and phenology of fine root endophyte colonization across populations of Lycopodiella inundata. Mycorrhiza 30(5): 577-587.

4. Omar, M. B., Bolland, L. and Heather, W. A. (1978). A permanent mounting medium for fungi. StainTechnol 53(5): 293-294.

5. Phillips, J. M. and Hayman, D. S. (1970). Improved procedures for clearing roots and staining parasitic and vesicular-arbuscular mycorrhizal fungi for rapid assessment of infection. $T$ Brit MycolSoc 55(1): 158-IN118.

6. Priyadharsini, P., Pandey, R. and Thangavelu, M. (2012). Arbuscular mycorrhizal and dark septate fungal associations in shallot (Allium cepa L. var. aggregatum) under conventional agriculture. Acta Botanica Croatica 71: 159-175.

7. Thangavelu, M. and Muthuraja, R. (2016). Arbuscular mycorrhizal and dark septate endophyte fungal associations in Asparagus. Turk J Bot 40: 662-675.

8. Vierheilig, H., Coughlan, A. P., Wyss, U. and Piché, Y. (1998). Ink and Vinegar, a Simple Staining Technique for Arbuscular-Mycorrhizal Fungi. Appl Environ Microb 64(12): 5004-5007.

9. Vierheilig, H., Schweiger, P. and Brundrett, M. (2005). An overview of methods for the detection and observation of arbuscular mycorrhizal fungi in roots. Physiol Plantarum 125(4): 393-404. 
10. Wilkes, T. I., Warner, D. J., Edmonds-Brown, V., Davies, K. G. and Denholm, I. (2019). $\underline{A}$ comparison of methodologies for the staining and quantification of intracellular components of arbuscular mycorrhizal fungi in the root cortex of two varieties of winter wheat. Access Microbiology 2(2). 